\title{
Age, Sex and Seasonal Variations of Dermatosis Attending in Dermatology Department of A Tertiary Care Hospital
}

\author{
Shamsun Nahar Binte Mannan ${ }^{\text {* }}$ \\ Md Zahangir Alam ${ }^{2}$ \\ Rajat Sanker Roy Biswas ${ }^{3}$
}

'Department of Dermatology Chattagram Maa Shishu-O-General Hospital Chittagong, Bangladesh.

${ }^{2}$ Department of Physical Medicine Chattagram Maa-O-Shishu Hospital Medical College Chittagong, Bangladesh.

${ }^{3}$ Department of Medicine

Chattagram Maa-O-Shishu Hospital Medical College Chittagong, Bangladesh.

\begin{abstract}
Background: Dermatological problems are common among patients of different ages visiting the out patient departments of tertiary care centers. So the objective of the study to see the spectrum of pediatric and adult dermatoses in out patient skin department of Chattagram Maa hishu O General Hospital. Methods: The medical records of children and adult attending the dermatology outpatient clinics of Chattagram Maa-O-Shishu Hospital for the year September 2015 to September 2016 were retrieved. Data of 8534 patients were included. Demographic data (Age and sex) and diagnoses were collected and analyzed. Results: Among 8534 patients attended in OPD, majority of the patients were female and children $(2500,56 \%$ \&, $3622,42 \%)$. Infection constituted most of the dermatosis $(31 \%)$ of which scabies was found most common (46\%), eczema comes after (20\%) and contact dermatitis prevailed (18\%). Regarding seasonal variations majority of the patient peaked in summer 855 (10\%). Among all 14 patients of epidermolysis bullosa were found. Leprosy and skin tuberculosis were found. Rare diseases including epidermal nevus syndrome 1 case, tuboeruptive xanthoma in children, 2 cases, subcutaneous fat necrosis in neonate -2 cases, atypical presentation of erythema multiforme were found. Conclusion : Many children and almost all adult presents with uncommon presentation of common disease due to frequently visiting non professional and traditional medicine practitioners and homeopathic and traditional medicine.
\end{abstract}

Key words: Dermatosis; Dermatology; OPD.

\section{INTRODUCTION}

Pattern and presentations of dermatological diseases differs in different countries and within various regions of a country depending on different factors like social, economic, racial and environmental. According to WHO, prevalence of skin diseases in general population is $(21-87 \%)^{1}$. Skin diseases are usually chronic with high morbidity and low mortality like eg. Psoriasis, Parapsoriasis, Darier's disease, but sometimes it may be fatal like autoimmune bullous diseases Pemphigus. A study done in India found that The profile of skin diseases varies widely among different geographical locations and over time. The patients attending the Dermatology outdoor of their hospital mostly are the under privileged urban slum dwellers, more than $85 \%$ of whom are below poverty line. It's a common knowledge that type and amount of disease in any community are affected directly or indirectly by climate. Various climatic factors that may determine the incidence of skin diseases are cold, heat, light, sunlight and humidity ${ }^{2}$. Also, different degrees of exposure to external factors as well as different levels of functional development of skin may give rise to differential prevalence of dermatoses among infants, toddlers and children. In the present study, it was tried to quantify and assess the significance of seasonal variation and trend of change with age of common dermatoses among the patients visiting our outdoor. 


\section{MATERIALS AND METHODS}

It was an observational study done during a one year period from September 2015 to August 2016. Prior hospital permission was taken from the authority for the study. Data are kept in a record book at dermatology outdoor. The medical records of children and adult attending the dermatology outpatient clinics of Chattagram Maa-O-Shishu Hospital for the year were retrieved. Here data of 8534 patients were included. Demographic data (Age and sex) and diagnoses were collected and analyzed. Data were compiled and analyzed by Microsoft Excel.

\section{RESULTS}

Among 8534 patients attended in OPD, majority of the patients were female and children $(2500,56 \% \&, 3622,42 \%)$. Infection constituted most of the dermatosis (31\%) of which scabies was most common (46\%) eczema comes after $(20 \%)$ and contact dermatitis prevailed (18\%). Age distribution revealed most of them are adult age group (51\%). Regarding seasonal variations majority of the patient peaked in summer 855 (10\%). Regarding neonatal dermatosis, 14 patients found having epidermolysis bullosa.

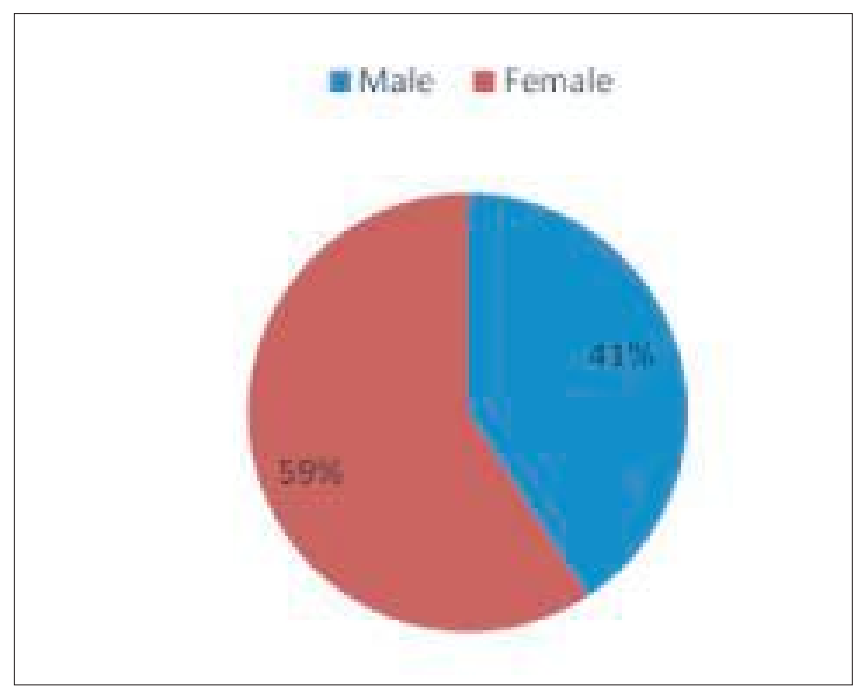

Figure 1 : Sex distribution.

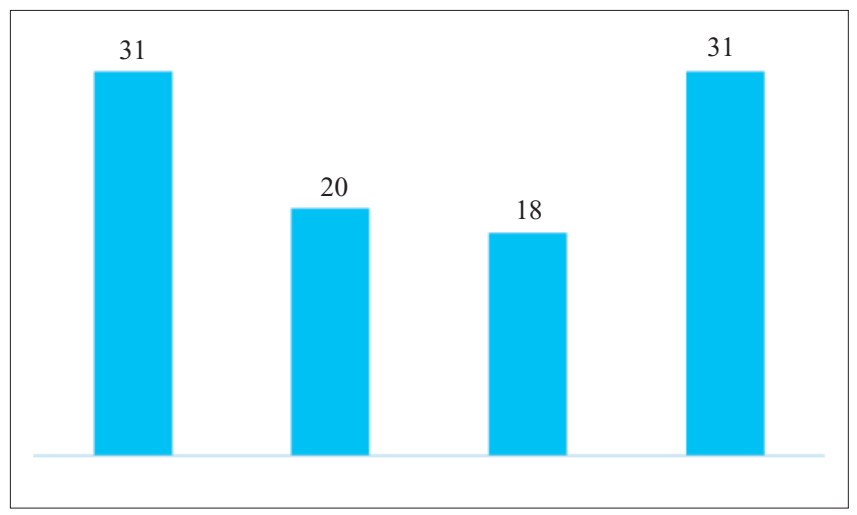

Figure 2 : Disease pattern.

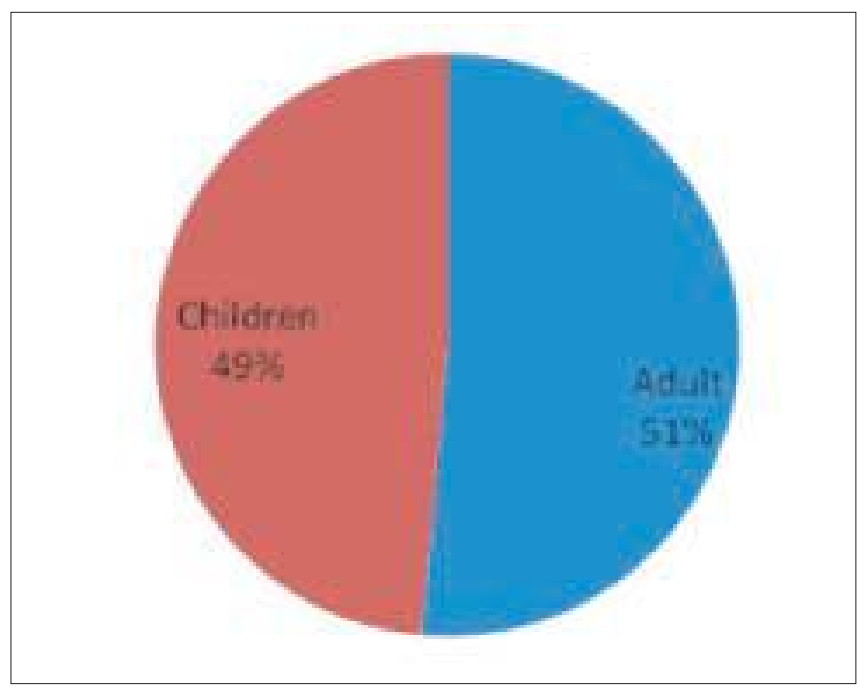

Figure 3 : Age variations.

Table 1 : Seasonal variations of dermatosis.

\begin{tabular}{llr} 
Season & Disease & Number (\%) \\
Summer & Fungal disease & $450(5.2 \%)$ \\
& Miliaria rubra & $175(2.01 \%)$ \\
\multirow{3}{*}{ Winter } & Eczema & $1426(16.7 \%)$ \\
& HZ & $51(0.5 \%)$ \\
& Measles & $14(0.16 \%)$ \\
& Molluscum contagiosum & $18(0.21 \%)$ \\
& Genital herpes & $1890.21 \%)$ \\
& Genodermatosis & $78(0.91 \%)$ \\
& Viral warts & $90(1.05 \%)$ \\
\hline
\end{tabular}

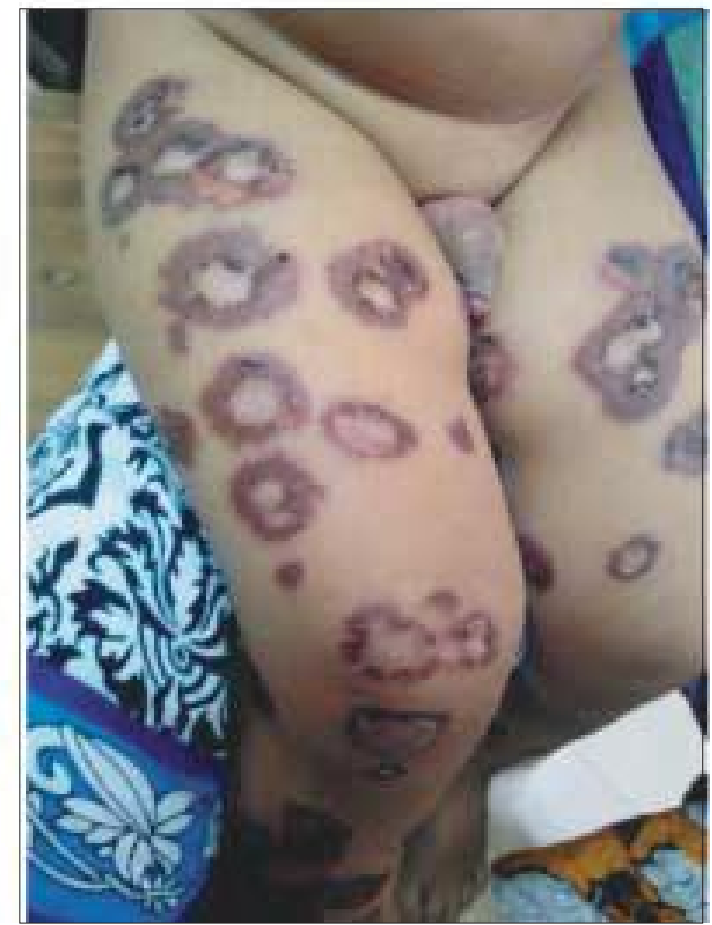

Figure 4 : Erythema multiforme. 


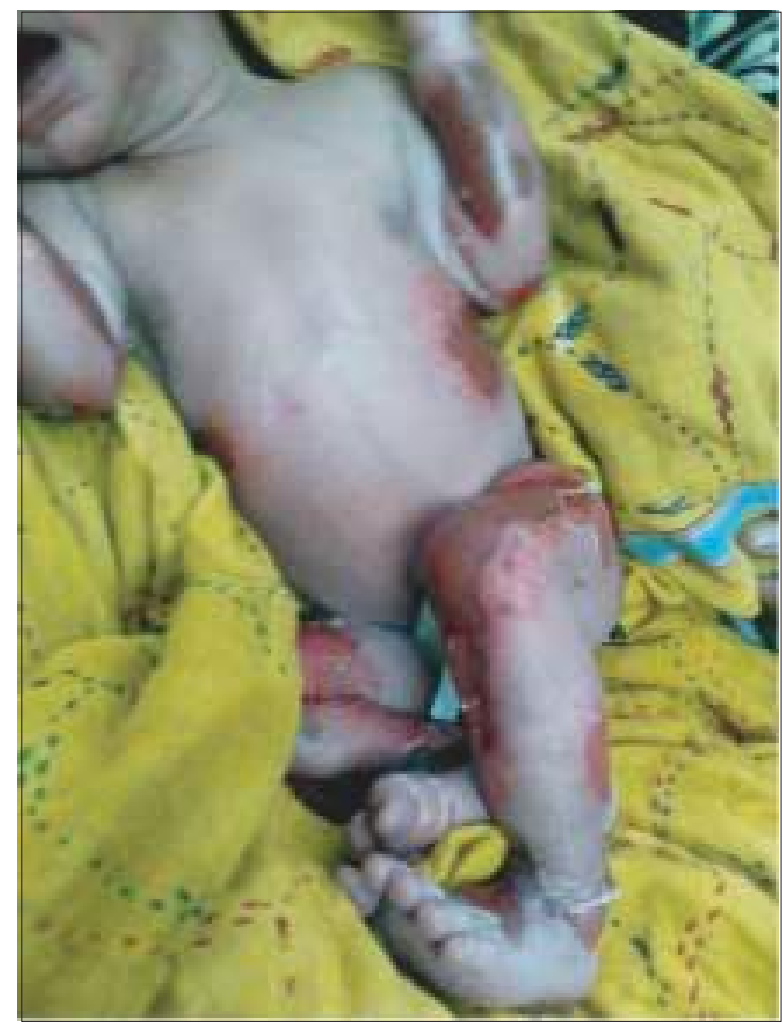

Figure 5 : Epidermolysis bulosa.

\section{DISCUSSION}

In our study among 8534 patients attended in OPD, we found majority were female and children $(2500,56 \% \&, 3622,42 \%)$. Children and females are the most vulnerable group in our society and they seek attention more. Age distribution revealed prevalence of bacterial infections and scabies, prurigo simplex in infants and preschool children, parasitic infections in school children and sebaceous gland disorders in adolescents. This may be attributed to the exposure to external environmental factors away from the cozy protection of home as well as increased physical contact with neighbours as the baby learns to walk. The finding also emphasized the high degree of parental care of an infant usually commands. Later, diminution of prevalence of miliaria as the toddlers reach the pre-schoolchild age group may be due to development of some degree of tolerance to the environmental factors, the so called 'hardening' of the $\operatorname{skin}^{3}$.
Infection constituted most of dermatosis (31\%) of which scabies was most common (46\%) eczema comes after (20\%) and contact dermatitis prevailed (18\%). Scabies and fungal infections were the most common in both genders. In our study, scabies most frequently affected the abdomen of males and upper limbs (Especially front of wrist and antecubital fossa) of females. Scalp was the second most common site of involvement after face in case of impetigo as well as furunculosis. This high degree of localization of bacterial infection to scalp is presumably due to the habit of applying oil on scalp and hair which is rampant in the community under study ${ }^{4}$.

Regarding seasonal variations majority of the patient peaked in summer $855(10 \%)$. Scabies prevailed in winter and spring, whereas bacterial infections, fungal infections, miliaria rubra pevailed in summer and autumn. Most dermatosis peaked in summer and autumn except urticaria and chicken pox peaked in spring.

Neonatal Genodermatosis are not uncommon.14 patients of epidermolysis bullosa were found. Leprosy and skin tuberculosis were found.Rrare diseases include epidermal nevus syndrome 1 , tuboeruptive xanthoma in children- 2 cases, subcutaneous fat necrosis in neonate- 2 cases ,atypical presentation of erythema multiforme were found due to misdiagnosis and maltreatment.

Many children and almost all adult present uncommon presentation of common disease due to frequently visiting non professional and traditional medicine practitioners and homeopathics and traditional medicine.

Infections and maltreatment with homeopathic and traditional medicine and malpractice of quack doctors and frequent use of steroid in any skin disease are parallel to the situation in developing communities. Such diseases and misshapes are potentially controllable and therefore strategies that target infections may represents a key to an efficient child health care programme.

\section{CONCLUSION}

In conclusion we hope that the study of distribution of lesions of common dermatoses will help to diagnose difficult cases and evaluate extensiveness of the disease by reminding us of the body parts which, by virtue of being commonly affected, are must-examine sites in young children of pediatric age group in our context.

\section{DISCLOSURE}

All the authors declared no competing interest.

\section{REFERENCES}

1. Bijayanti DT, Zamzachin G. Pattern of skiin diseases in Imphal. Indian J Pediatr. 2006; 51: 149-150.

2. Handa H, Handa S, Handa R. Environmental factors and the skin. In: Valia RG, Valia AR, editors. IADVL Textbook and Atlas of Dermatology. 2nd ed. Mumbai, India: Bhalani. 2001;82-92.

3. Ghosh SK, Saha DK, Roy AK. A clinico-aetiological study of dermatoses in pediatric age group. Ind J Dermatol. 1995;40:29-31.

4. James WD, Berger TD. Elston DM. eds. Andrew's diseases of the skin: clinical dermatology. $10^{\text {th }}$ ed. Philadelphia: Elsevier Saunders. $2006 ; 220$. 